\title{
Applications and Perspectives of Biobased Oligomeric Esters in the Industry
}

\section{Utsunomia $C^{*}$}

Institute of Life Technologies, University of Applied Sciences and Arts Western, Switzerland

*Corresponding author: Camila Utsunomia, University of Applied Sciences and Arts Western Switzerland (HES-SO Valais-Wallis), Institute of Life Technologies, Sion, Switzerland; Email: camila.utsunomia@hevs.ch

\section{Mini Review \\ Volume 5 Issue 1}

Received Date: March 06, 2020

Published Date: March 16, 2020

DOI: 10.23880 /oajmb-16000159

\section{Abstract}

Our society has the imperative need to reduce the production, utilization, and pollution caused by petroleum-based materials. In addition to the biopolymers, such as polylactide (PLA) and polyhydroxyalkanoates (PHA), biobased oligoesters having a different set of properties and applications are yet to be explored as alternatives to materials derived from finite carbon sources. This mini-review highlights the potential applications of biobased oligoesters synthesized via chemical, enzymatic, and biological routes.

Keywords: Oligomers; Renewable; Molecular Weight; Biopolyester; Medical

\section{Abbreviations: PCL: Polycaprolactones; PEA:} Polyesteramides; PHA: Polyhydroxyalkanoates; PLA: Polylactide; D-LAOs: Lactate-Based Oligomers; DEG: Diethylene Glycol.

\section{Introduction}

Plastics are ubiquitous materials which became indispensable to the modern society since mid-1950 [1]. These organic polymers are composed of repetitive monomer units and have high molecular weights (typically thousands to hundreds of thousands of Daltons for synthetic plastics in the market [2]). The vast majorities of commercial polymers is produced from petroleum and its derivatives and are widely used in the construction, medical, electrical, automobile, agriculture, and food industries [3]. Packaging, particularly, is the largest market of plastics which have grown towards the fabrication of single-use goods [1]. Essentially, synthetic plastics are not biodegradable, with few exceptions such as polycaprolactones (PCL), polyesteramides (PEA), aliphatic copolyesters (e.g. polybutylene succinate adipate), and aromatic copolyesters (e.g. polybutylene adipate terephthalate) [4]. The upscaling concerns on water and soil pollution as well as the intoxication of humans [5] and animals caused by the overwhelming environmental contamination of petroleum-based plastics are driving the research and implementation of more sustainable alternatives to these materials.

Synthesis of biobased and biodegradable/compostable materials are, therefore, of huge interest to decrease the humankind dependency and mitigate the detrimental effects of synthetic plastics. Examples of biopolymers are polysaccharides, proteins, lipids, the class of microbial polyesters polyhydroxyalkanoates (PHA), and polylactide (PLA) [4]. Biopolymers have been extensively studied and some of them, such as PLA led by NatureWorks LLC, are expanding the market participation along the years [6]. Due to the increasing environmental consciousness and compulsory need of reducing synthetic materials utilization, production scale-up and commercialization of biobased and biodegradable materials are expected to grow in the next decades.

With less attention given, biobased oligomeric esters or oligoesters, on the other hand, could fulfill another range of needs from the industry and further diminish the use of nonrenewable carbon sources. These oligomers, which are the low molecular weight counterparts of polymers, depending on the type of monomer can present low melting points 


\section{Open Access Journal of Microbiology \& Biotechnology}

and are mostly liquid/semi solid, in contrast to the solid polymers, at room temperature $[7,8]$. Therefore, biobased oligoesters find potential to be applied in the formulation of drug delivery carriers, plasticizers, soft tissue implants, green solvents, cosmetics, and building blocks for polymer synthesis, such as tailor-made block copolymers [7]. Chemical, enzymatic, and in vivo synthesis of oligoesters and their possible applications are exemplified below.

Synthesis of oligomers from plant sugars-based 1, 3-propanediol and succinic acid was designed to generate new materials for personal care and cosmetic formulations (e.g., as film-formers and structuring agents) [9]. Alcohol terminated oligomeric propylene succinate were obtained in a combination of solution and melt-phase condensation polymerization in toluene at $130-150{ }^{\circ} \mathrm{C}$. The weightaveraged molecular weights were around 900,1800, and $2700 \mathrm{Da}$. Afterwards, fatty acids were conjugated to the oligomers. Materials ranging from amorphous oils to semicrystalline solids were obtained by varying the molecular weight and the fatty acid end-groups.

A bio catalytic route for the synthesis of oligoesters of plant-based hydroxy-fatty acids and $\varepsilon$-caprolactone was investigated [10]. Among the fatty acids, only 10-hydroxystearic acid was synthesized using cell-free extract of recombinant Escherichia coli expressing a gene encoding oleate hydratase. Immobilized lipases were used as catalysts and reactions were carried out using toluene as solvent at temperatures between $45-85^{\circ} \mathrm{C}$ for the bio catalytic synthesis of linear, branched, and cyclic oligoesters. The obtained oligomers can be further functionalized and applications on drug-delivery systems and bioresorbable scaffolds are yet to be demonstrated.

Oligomers of PHAs were produced and secreted by a recombinant $E$. coli heterologously expressing the genes, particularly a mutant PHA synthase, for the production of lactate-based oligomers (D-LAOs). The synthesis is significantly improved when diethylene glycol (DEG) is supplemented in the bacterial culture medium. The result are functionalized D-LAOs with DEG at the carboxyl termini. These oligoesters are produced from plant sugars (e.g., glucose and xylose) and can be utilized, but not only, as building blocks for the synthesis of LA-based polymers and poly(ester-urethane)s [11].

The physical properties of oligoesters can be tailored by varying the monomer type, introducing functional groups or promoting copolymerization, combining with other materials, and fine-tuning the molecular weight. Therefore, these oligomers can have a wide range of applications from ordinary to value-added ends. The most important challenges to make biobased oligoesters competitive include to controlling the synthesis to meet the required material properties, scale-up the production, and reduce the synthesis costs.

\section{References}

1. Geyer R, Jambeck JR, Law KL (2017) Production, use, and fate of all plastics ever made. Sci Adv 3(7): e1700782.

2. Llorens J, Rude E, Marcos RM (2000) Unimodal molecular weight distribution of commercial polymers from viscoelastic data. Journal of Polymer Science Part B: Polymer Physics 38(11): 1539-1546.

3. Singh N, Hui D, Singh R, Ahuja IPS, Feo L, et al. (2017) Recycling of plastic solid waste: A state of art review and future applications. Composites Part B: Engineering 115: 409-422.

4. Vieira MGA, Beppu MM, Altenhofen da Silva M, Oliveria dos Santos L (2011) Natural-based plasticizers and biopolymer films: A review. European Polymer Journal 47(3): 254-263.

5. Wright SL, Kelly FJ (2017) Plastic and human health: A Micro issue? Environ Sci Technol 51(12): 6634-6647.

6. Vink ET, Davies S (2015) Life Cycle Inventory and Impact Assessment Data for 2014 Ingeo $^{\mathrm{TM}}$ Polylactide Production. Industrial Biotechnology 11(3): 167-180.

7. Piromjitpong P, Kiriratnikom J, Ungpittagul T, Yotphan, Phomphrai K (2018) Rapid one-pot catalytic synthesis of double-capped oligoesters from lactones. Applied Catalysis A: General 549: 134-140.

8. Amsden B, Hatefi A, Knight D, Bravo Grimaldo E (2004) Development of biodegradable injectable thermoplastic oligomers. Biomacromolecules 5(2): 637-642.

9. Hackenberg JD, Stebbins ND, Uhrich KE (2017) Biobased oligomeric esters comprised of 1,3-propanediol, succinic Acid, and fatty Acids. Macromolecular Chemistry Physics 218(20): 1700190.

10. Todea A, Aparaschivei D, Badea V, Boeriu CG, Peter F (2018) Biocatalytic Route for the Synthesis of oligoesters of hydroxy-fatty acids and $\epsilon$-caprolactone. Biotechnol J 13(6): e1700629.

11. Utsunomia C, Taguchi S (2018) Microbial secretion system of lactate-based oligomers and its application. Green Polymer Chemistry: New Products, Processes, and Applications (Chapter 4) ACS Publications, pp: 41-60.

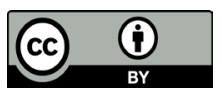

